\title{
Magnetic field effect on the photooxidation efficiency of ferrocene ${ }^{1}$
}

\author{
P. Gilch ${ }^{2}$, M. Linsenmann, W. Haas ${ }^{3}$, U.E. Steiner \\ Fakultät für Chemie, Universität Konstanz, D-78434 Konstanz, Germany
}

\begin{abstract}
The cage escape efficiency $\eta_{\mathrm{ce}}$ of radicals in the electron transfer quenching of triplet methylene blue by ferrocene in acetonitrile was found to be sensitive towards an external magnetic field. The field dependence of the effect corresponding to a monotonic decrease of $\eta_{\mathrm{ce}}$ was determined in a field range from 0.0 to $3.2 \mathrm{~T}$, where it reaches a value of $-20.7 \%$. The effect is analyzed in terms of a spin chemical model wherein the effective rate of geminate reverse electron transfer, regenerating the singlet ground state of the reactants, becomes magnetic field dependent due to magnetic mixing of non-uniformly reactive spin sublevels by the anisotropic Zeeman interaction. From the analysis, the absolute values of the rate constants of spin-allowed reverse electron transfer $\left(605 \mathrm{~ns}^{-1}\right)$, cage escape $\left(15 \mathrm{~ns}^{-1}\right)$, and electron spin relaxation in the ferricenium cation $\left(154 \mathrm{~ns}^{-1}\right)$ could be determined.
\end{abstract}

\section{Introduction}

Magnetic field effects on chemical reaction kinetics are due in most cases to some variant of the so-called radical pair mechanism [1] wherein diffusion, spin selective reactions and spin motion are combined into an intriguing reaction scenario [2] with spin correlated radical pairs (RPs) functioning as reaction intermediates [3]. The principle of spin conservation during an elementary chemical reaction step provides the basis of spin selectivity for a particular (usually the singlet) radical pair substate.

\footnotetext{
${ }^{1}$ Dedicated to Professor H.E.A. Kramer on the occasion of his 60 th birthday.

${ }^{2}$ Present adress: Institut für Physikalische Chemie, TU München, Lichtenbergstraße 4, D-85748 Garching.

${ }^{3}$ Present adress: Lawson Mardon Singen GmbH, AlusingenPlatz 1, D-78224 Singen.
}

Spin processes interconverting the four RP spin substates can therefore control the overall reactivity of the RP. It is through the effect an external magnetic field exhibits on the electron spin motion in the radicals of the pair that it can influence the kinetics and yields of such reactions.

The quantitative characteristics of the magnetic field effects are largely determined by the types of interaction that govern the intrinsic dynamics of spin motion. If it is a hyperfine interaction, as is the case in the classical areas of spin chemistry (chemically induced nuclear and electron spin polarization: CIDNP, CIDEP, and the magnetic isotope effects: MIE) the magnetic field dependence is saturated at rather low fields up to several hundreds of gauss. What can be learned from such experiments refers to the energetics of rather weak interactions and to kinetic processes with time constants down to some nanoseconds. In recent work about magnetic field 
effects on the photooxidation of $\mathrm{Ru}^{\mathrm{II}}$-trisdiimine complexes [4-8] we have shown that the concepts of the radical pair mechanism and the pertinent spin formalism can be generalized to systems with heavily perturbed spin states, namely to metal complexes with strongly spin-orbit coupled Kramers doublets from open-shell d-electron configurations. These systems require high magnetic fields to be affected but, in turn, evaluation of the magnetic field dependence affords access to reaction time constants and spin relaxation times in the ps time domain.

In this Letter we report a successful attempt to extend this type of d-electron spin chemistry to radical pairs involving the ferricenium ion, another most interesting case of a $\mathrm{d}^{5}$ electron system.

For our investigations we resorted to the photooxidation of ferrocene $(\mathrm{Fc})$ by the excited triplet of methylene blue $\left({ }^{3} \mathrm{MB}^{+}\right)$a reaction that was first investigated by Farmilo and Wilkinson [9] and later studied in more detail by Kikuchi et al. [10] who focussed on a quantitative determination of the efficiency of free radical formation determined by the following processes:

$$
\begin{aligned}
& \mathrm{Fc}^{3}{ }^{3} \mathrm{MB}^{+} \stackrel{k_{\mathrm{q}}}{\rightarrow}{ }^{3}\left(\mathrm{Fc}^{+} . . \mathrm{MB}^{\prime}\right), \\
& { }^{3}\left(\mathrm{Fc}^{+} . . \mathrm{MB}\right) \stackrel{k_{\text {isc.bct }}}{\rightarrow} \mathrm{Fc}^{\circ . \mathrm{MB}^{+},} \\
& { }^{3}\left(\mathrm{Fc}^{+} . . \mathrm{MB}\right) \stackrel{k_{\mathrm{cc}}}{\rightarrow} \mathrm{Fc}^{+}+\mathrm{MB}^{\prime},
\end{aligned}
$$

i.e. reductive quenching of ${ }^{3} \mathrm{MB}^{+}$by electron transfer from $F c$ producing a charge shifted state ${ }^{3}\left(\mathrm{FC}^{+} . . \mathrm{MB}\right)$ which may dissociate into free radicals or undergo geminate reverse electron transfer to regenerate the ground state reactant. Kikuchi et al. considered the primary product as a triplet exciplex. Although the spin-forbidden nature of the reverse electron transfer from this species was recognized, the rate constant $k_{\text {isc,bct }}\left(k_{\text {isc }}\right.$ in their notation) was considered to be fast due to the strong spin-orbit coupling at the $\mathrm{Fe}$ centre. In the quenching experiments only the rate constant $k_{\mathrm{q}}$ could be directly determined. The absolute values of the rate constants $k_{\text {isc.bet }}$ and $k_{\text {ce }}$ were not accessible but their ratio could be assessed from the efficiency $\eta_{\mathrm{ce}}$ of free radical formation (cage escape). In fact, the value of 0.21 found for $\eta_{\text {ce }}$ confirms the high rate of reverse electron transfer.
As we have shown in our previous investigations of Ru-complex spin chemistry [4-8], in metal centered d-electron radicals the rate of spin processes may be comparable to that of fast electron transfer processes, so that magnetic modulation of the spin processes will lead to detectable kinetic effects from which the details of the geminate kinetics can be determined. Here we will show that this method is also fully applicable to the case of the $\left(\mathrm{Fc}^{+} . . \mathrm{MB}\right)$ system.

\section{Experimental}

Ferrocene (Fluka) was recrystallized twice from ethanol. Methylene blue from the stock of the laboratory was purified by an extraction method according to the method of Bergmann and O'Konski [11]. The sample purity was checked by $\mathrm{CHN}$ analysis, thin layer chromatography and absorption spectroscopy $\left(\lambda_{\max }=650 \mathrm{~nm}, \epsilon_{\max }=84500 \mathrm{M}^{-1} \mathrm{~cm}^{-1}\right.$ in methanol). Acetonitril (UVASOL from Merck) was the solvent used for the experiments. Sample solutions were deaerated by flushing them with suprapure nitrogen (Messer-Griesheim, 5.0).

Nanosecond laser flash photolysis experiments utilizing a flow-through system with the flow-through sample cuvette mounted between the pole pieces of a Bruker B-E 15 electromagnet were carried out with the equipment described in ref. [12], however replacing the excimer pumped dye laser by a Nd-YAG laser (Quanta Ray GCR 150-5) operated at its second harmonic $(532 \mathrm{~nm})$. The pulse width was 5-7 ns. The laser energy was attenuated to $30 \mathrm{~mJ}$ per pulse. All measurements were done at room temperature.

\section{Results and discussion}

\subsection{Transient absorption kinetics and magnetic field effect}

Exciting methylene blue solutions with the laser pulse at $532 \mathrm{~nm}$ yields a transient absorption spectrum in good agreement with that described for ${ }^{3} \mathrm{MB}^{+}$in refs. [13,14]. The addition of ferrocene, which itself has negligible absorption at the laser 


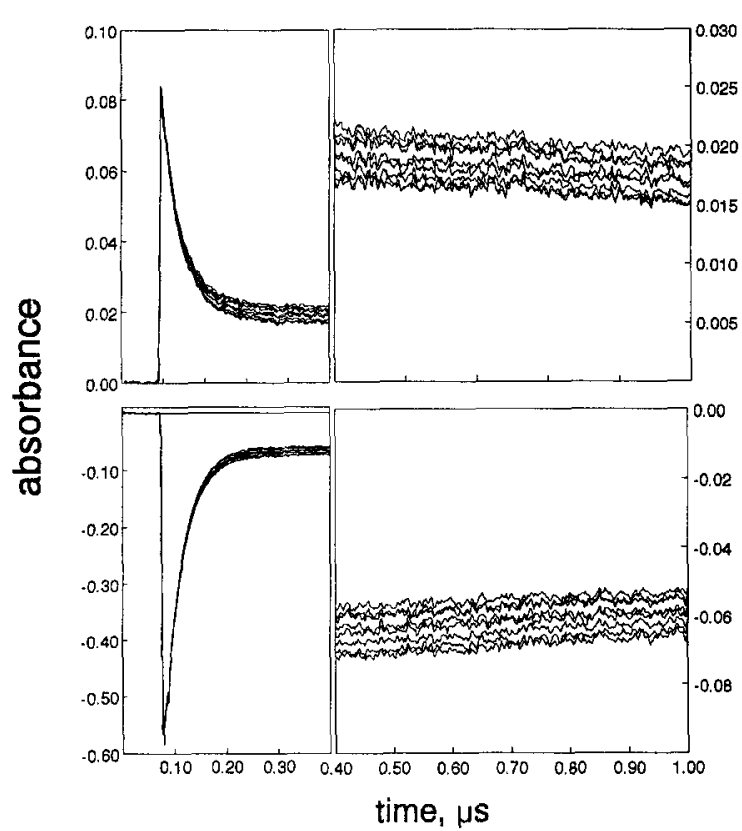

Fig. 1. Laser flash induced transient absorption signals from an acetonitrile solution of methylene blue $(10 \mu \mathrm{M})$ and ferrocene $(0.2 \mathrm{mM})$ excited at $532 \mathrm{~nm}$. The signals were recorded with the following magnetic field settings: $0.0,0.5,1.0,1.5,2.0,2.5,3.0$, $3.2 \mathrm{~T}$. Top: observed at $430 \mathrm{~nm}$, order of fields from top to bottom. Bottom: observed at $654 \mathrm{~nm}$, order of fields from bottom to top. On the right the absorbance scale is enlarged.

wavelength, leads to a shortening of the triplet decay corresponding to a quenching rate constant of $1.3 \times$ $10^{10} \mathrm{M}^{-1} \mathrm{~s}^{-1}$ in good agreement with the value of $1.1 \times 10^{10} \mathrm{M}^{-1} \mathrm{~s}^{-1}$ reported by Kikuchi et al. [10]. As a result of the quenching, a long-lived absorption characteristic of semireduced $\mathrm{MB}$ radical appears, providing evidence for the electron transfer nature of the quenching reaction. For a quantitative determination of the efficiency of free radical formation and its magnetic field dependence the transients were monitored at three wavelengths:

- $825 \mathrm{~nm}$, the first absorption maximum of the T-T absorption of ${ }^{3} \mathrm{MB}^{+}$,

- $654 \mathrm{~nm}$, the absorption maximum of the $\mathrm{MB}^{+}$ $S_{0} \rightarrow S_{1}$ absorption in acetonitrile and

- $430 \mathrm{~nm}$, the absorption maximum of $\mathrm{MB}$, and also close to the second absorption maximum of ${ }^{3} \mathrm{MB}^{+}$.

Signals at 654 and $430 \mathrm{~nm}$ are shown in Fig. 1. For these signals the concentration of $\mathrm{Fc}$ was high enough to ensure that the contribution of deactiva- tion channels of ${ }^{3} \mathrm{MB}^{+}$other than quenching by $\mathrm{Fc}$ is negligible. Triplet quenching is completed after about $0.2 \mathrm{~s}$. In the time window shown in the Figure the long-lived absorption exhibits only a slight decay which is due to the homogenous recombination reaction of $\mathrm{Fc}^{+}$and $\mathrm{MB}$. As exhibited by the transient signals, particularly at $654 \mathrm{~nm}$, where ground state bleaching is seen, the yield of free radicals is much less than 1. Using the absorption coefficients given by Kikuchi et al. for the ${ }^{3} \mathrm{MB}^{+}$triplet at $825 \mathrm{~nm}$ and for the radical at $430 \mathrm{~nm}$, our measurements yielded an efficiency $\eta_{\mathrm{ce}}=0.20$ of free radical formation from the triplet which compares well with the value of 0.21 reported by Kikuchi et al. [10].

As can be clearly seen from Fig. 1, the yield of free radicals (but not the triplet formation yield and the triplet quenching rate constant) is sensitive to a magnetic field. Increasing the field, reduces the free radical yield which is consistent with an acceleration of the overall rate constant of geminate reverse electron transfer by a magnetic field. Within experimental accuracy the field effects exhibited by the long-lived transients at 654 and $430 \mathrm{~nm}$ are the same. Due to a higher extinction coefficient of the ground state at $654 \mathrm{~nm}$ as compared to that of the radical at $430 \mathrm{~nm}$ the former signal shows a somewhat better signal to noise ratio. Therefore the signals at $654 \mathrm{~nm}$ were used for a quantitative determination of the magnetic field effect. This determination was based on a simulation of the ground state recovery kinetics using the relation

$\Delta A=\left(\Delta A_{0}-\Delta A_{\infty}\right) \exp \left(-k_{\text {eff }} t\right)+\Delta A_{\infty}$,

where $\Delta A_{0}$ is the transient absorbance directly after the laser pulse, $k_{\text {eff }}$ is the effective first order decay constant and $\Delta A_{\infty}$ is proportional to the concentration of free $\mathrm{MB}$ radicals having escaped the geminate recombination process. In order to reduce data scatter caused by variations in laser intensity, all $\Delta A_{\infty}$ were normalized by their corresponding $\Delta A_{0}$ values:

$a_{\infty} \equiv \frac{\Delta A_{\infty}}{\Delta A_{0}}$.

Using the normalized $a_{\infty}$ values, we define the relative magnetic field effect $R(B)$ on $\eta_{\mathrm{ce}}$ as:

$R(B)=\frac{a_{\infty}(B)-a_{\infty}(0)}{a_{\infty}(0)}$. 


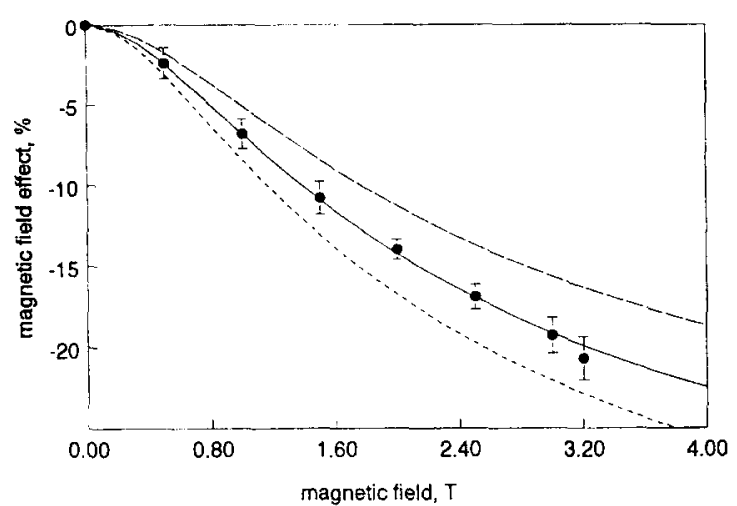

Fig. 2. Relative magnetic field effect (defined in Eq. (6)) on the efficiency of free radical formation in the reaction of ${ }^{3} \mathrm{MB}^{+}$with ferrocene. The lines correspond to theoretical simulations with $k_{c e}=1.5 \times 10^{10} \mathrm{~s}^{-1}$ and $\tau_{\mathrm{s}}=7.5 \mathrm{ps}(-.-), 6.5 \mathrm{ps}(\longrightarrow)$ and 5.5 ps (_- - ) , respectively. The $k_{\mathrm{bct}}$ values $(687,605,519$ $\mathrm{ns}^{-1}$, respectively) were adjusted so as to maintain a constant value of $\eta_{\text {ce }}=0.21$ at zero field.

In Fig. 2 details of the magnetic field dependence of $\eta_{\mathrm{ce}}$ are shown.

\subsection{Analysis of the magnetic field effect}

\subsubsection{The spin chemical model}

The spin chemical model applied here was originally introduced to account for the MFE in Ru(III)complex/methylviologen radical pairs. It is described in detail in refs. $[5,6]$. Here we give just a short summary.

Spin dynamics, chemical and motional kinetics of the radical pair are described by a stochastic Liouville equation,

$$
\frac{\mathrm{d} \rho}{\mathrm{d} t}=-\frac{\mathrm{i}}{\hbar}[H, \rho]_{-}+\mathfrak{R} \rho-\frac{1}{2}[K, \rho]_{+},
$$

for the spin density matrix $\rho$ of the pair represented in the following coupled basis of effective doublet spin states of the individual radicals:

$$
\begin{aligned}
& S^{\prime}=\frac{1}{\sqrt{2}}\left(\alpha \beta^{\prime}-\beta \alpha^{\prime}\right), \\
& T_{0}^{\prime}=\frac{1}{\sqrt{2}}\left(\alpha \beta^{\prime}+\beta \alpha^{\prime}\right), \\
& T_{+}^{\prime}=\alpha \alpha^{\prime}, \\
& T_{-}^{\prime}=\beta \beta^{\prime} .
\end{aligned}
$$

Primed symbols are used where the effective spin states represent mixed spin-orbit states. Due to the spin-orbit mixed nature of the primed states the pair states defined in Eqs. (8-11) are not pure spin states. Nevertheless, this basis of states provides for the maximum possible concentration of singlet and triplet character on the individual states (cf. below). For the explicit representation of the operators in Eq. (7) we refer to refs. $[5,6]$. Here we shall only give a qualitative description and specify the parameters of the model. The spin Hamiltonian $H$ is a pure Zeeman Hamiltonian specified by two axial g-tensor components $g_{\|}$and $g_{\perp}$ of the complex and the isotropic $g$-factor of the organic radical. We note that in the basis given by Eqs. $(8-11)$ the axis of quantization is defined parallel to the $g_{\|}$axis of the complex. Zeeman interaction causes coherent mixing of the given basis states. This behaviour is analogous to the ordinary $\Delta g$-mechanism in radical pairs but more complex. The relaxation superoperator $\Re$ accounts for the effects of spin relaxation in the paramagnetic complex due to rotation-independent contributions parametrized by a spin relaxation time $\tau_{\mathrm{s}}$ and spin rotational coupling determined through the rotational diffusion coefficient $d_{\mathrm{r}}$ of the complex and its $\gamma$ tensor components [15]. It should be noted, though, that the rotational contribution is only of minor importance compared to the rotation-independent one. Spin relaxation in the organic radical is completely neglected.

The reaction channels of the radical pair are described within the exponential model. The reaction operator $k$ accounts for radical pair decay by reverse electron transfer and dissociation into free radicals. Whereas the latter, described by a rate constant $k_{\mathrm{ce}}$, is considered as spin-independent, the former process is spin selective for singlet multiplicity. This is accounted for by assigning individual reaction constants $p_{\mathrm{S}, i} k_{\mathrm{bct}}$ to the basis states, where $p_{\mathrm{S}, i}$ denotes the singlet character of substate $i$, and $k_{\mathrm{bct}}$ is the rate constant of a fully spin allowed reverse electron transfer.

The efficiency $\eta_{\text {ce }}$ of cage escape is easily obtained from the time integrated solution of Eq. (7) which is calculated numerically. Finally, the $\eta_{\text {ce }}$ values resulting for different complex orientations relative to the magnetic field are averaged over an isotropic orientational distribution. 
3.2.2. Singlet character of the radical pair's formal spin states

In $D_{5 d}$ symmetry notation (corresponding to a staggered geometry) for the molecular orbitals, the ground state of the $\mathrm{Fc}^{+}$radical is derived from an $\mathrm{a}_{1 \mathrm{~g}}^{2} \mathrm{e}_{2 \mathrm{~g}}^{3}$ configuration [16]. The resultant ${ }^{2} E_{2 g}$ state is split due to spin-orbit coupling and orthorhombic distortion [17] yielding two Kramers doublets. Using the hole formalism and neglecting MO contributions from the $\mathrm{Cp}$ rings the representation of the lower one of these doublets is:

$\alpha^{\prime}=\left(c \mathrm{~d}_{x^{2}-y^{2}}+\mathrm{i} s \mathrm{~d}_{x y}\right) \alpha$,

$\beta^{\prime}=\left(c \mathrm{~d}_{x^{2}-y^{2}}+\mathrm{is}_{x y}\right) \boldsymbol{\beta}$,

where $s$ and $c$ are sine and cosine, respectively, of some characteristic angle. They are related to the $g$-tensor components by:

$g_{\perp}=g_{c}\left(c^{2}-s^{2}\right)$,

$g_{\|}=g_{c}+8 \kappa c s$.

Here $\kappa$ is a reduction factor accounting for the quenching of orbital momentum by the mixing of d-orbitals with ligand MOs and for the effects of dynamic Jahn-Teller distortion [18], $g_{\mathfrak{e}}$ is the $g$-factor of the free electron.

It should be noted that although, according to Eqs. $(12,13)$, the representation of the Kramers doublet components is factorized into a space part and a spin part, giving the $\alpha^{\prime}$ state $\alpha$ pure character and the $\beta^{\prime}$ state pure $\beta$ character, the space parts are partially orthogonal, i.e. we need a two-dimensional basis for the orbital part, the consequence of which will be that the set of radical pair states given in Eqs. (8-11) are not fully transformable into a set of pure singlet and triplet substates. This consequence becomes evident when representing the pair states of Eq. (8-11) in a basis of pure pair spin states derived from the two configurations $\left(\mathrm{d}_{x^{2}-y^{2}} \pi\right)$ and $\left(\mathrm{d}_{x y} \pi\right)$ where $\pi$ stands for the $\pi$-MO of the electron acceptor radical. The configurational origin of the corresponding states, two triplets and two singlets with altogether eight spin components, will be signified by indices 1 and 2 , respectively. With these pure spin states the radical pair states are represented as:

$\mathrm{T}_{+}^{\prime}=c \mathrm{~T}_{1,+}+\mathrm{i} s \mathrm{~T}_{2,+}$,

$\mathrm{T}_{-}^{\prime}=c \mathrm{~T}_{1,-}+\mathrm{i} s \mathrm{~T}_{2,-}$,
$\mathrm{T}_{0}^{\prime}=c \mathrm{~T}_{1,0}-\mathrm{i} s \mathrm{~S}_{2}$,

$\mathrm{S}^{\prime}=\mathrm{i} s \mathrm{~T}_{2,0}+c \mathrm{~S}_{1}$.

As follows from these equations, $T_{0}^{\prime}$ and $S^{\prime}$ are necessarily of spin-mixed nature. While $\mathrm{T}_{+}^{\prime}$ and $\mathrm{T}_{-}^{\prime}$ are of pure triplet character, both singlet and triplet character are distributed over $\mathrm{T}_{0}^{\prime}$ and $\mathrm{S}^{\prime}$. Within the $\mathrm{T}_{0}^{\prime}, \mathrm{S}^{\prime}$ subset a complete singlet-triplet separation is not possible because two different electronic configurations are involved. The singlet character of these states is given by $s^{2}$ and $c^{2}$, respectively, and can be expressed as:

$p_{s, \mathrm{~T}_{0}^{\prime}}=0.5-\frac{g_{\perp}}{2 g_{\mathrm{e}}}$,
$p_{s, S^{\prime}}=0.5+\frac{g_{\perp}}{2 g_{\mathrm{e}}}$.

\subsubsection{Quantitative analysis of the observed magnetic field effect}

The following values for the $g$-tensor components of $\mathrm{Fc}^{+}$can be found in the literature: 4.35 [g12] and 4.4 [g14] for $g_{\|}, 1.26$ [g12] and 1.34 [g14] for $g_{\perp}$. For our calculations we used the rounded values of

$g_{\|}=4.4$ and $g_{\perp}=1.3$,

yielding from Eqs. $(20,21)$

$p_{s, \mathrm{~S}}=0.825$ and $p_{s, \mathrm{~T}_{0}^{\prime}}=0.175$.

Assuming that the initially pure triplet spin multiplicity of ${ }^{3} \mathrm{MB}^{+}$is conserved in the creation of the radical pair, the initial populations of the primed radical pair substates are assumed to be distributed according to their triplet character, viz.

$\mathrm{T}_{+}^{\prime}: 1 / 3 \mathrm{~T}_{-}^{\prime}: 1 / 3 \quad \mathrm{~T}_{0}^{\prime}: 0.825 / 3 \quad \mathrm{~S}^{\prime}: 0.175 / 3$.

The contribution of spin-rotational relaxation is taken into account with an estimated value of $D_{\mathrm{r}} \approx 7.5$ $\mathrm{ns}^{-1}$ for the rotational diffusion constant of $\mathrm{Fc}^{+}$and using the following relations that can be derived for the components of the $\gamma$-tensor [19]:

$\gamma_{\perp}=\frac{g_{\perp}}{2}=0.65, \gamma_{\|}=g_{\|}-1=3.4$.

The remaining free parameters of the model are $k_{\mathrm{ce}}$, $k_{\mathrm{bct}}$, and $\tau_{\mathrm{s}}$. One degree of freedom for choosing their values is lost by the necessity of reproducing 
the experimentally observed absolute value of $\eta_{\mathrm{ce}}=$ 0.21 (we use the probably more precisely determined absolute value of ref. [10] instead of our value of 0.20 which, however, has no significant effect on the parameter values finally obtained). Thus, to fit its observed magnetic field dependence we are left with only two degrees of freedom. It turns out that the experimental observations can be nicely reproduced within rather narrow bounds of about $10 \%$ for all of the three parameters. The best-fit values are:

$k_{\mathrm{ce}}=15 \mathrm{~ns}^{-1}, k_{\mathrm{bct}}=605 \mathrm{~ns}^{-1}$ and $\tau_{\mathrm{s}}=6.5 \mathrm{ps}$.

The good quality of the fit can be judged from Fig. 2 , where the sensitivity of the calculated magnetic field effect with respect to a variation of $\tau_{\mathrm{s}}$ is demonstrated. At least formally, this result provides strong evidence for the validity of our spin chemical model. More specifically, the model can be confirmed by comparing the spin chemical results with independent information available for the parameters $k_{\text {ce }}$ and $\tau_{s}$.

A value for the rate constant $k_{\text {ce }}$ of cage escape may be estimated from the Eigen-Debye expression [20] which in the absence of a Coulomb interaction is given by

$k_{\mathrm{ce}}=3\left(D_{1}+D_{2}\right) / r^{2}$,

with $D_{1}$ and $D_{2}$ the diffusion constants of the reactants and $r$ their distance in the reaction complex. In acetonitrile, the diffusion constants of $\mathrm{Fc}$ and $\mathrm{MB}^{+}$are $2.18 \times 10^{-5} \mathrm{~cm}^{2} \mathrm{~s}^{-1}$ [21] and $1.1 \times$ $10^{-5} \mathrm{~cm}^{2} \mathrm{~s}^{-1}[19,22]$, respectively. Assuming the same values for the corresponding radicals and applying the Stokes-Einstein relation

$D_{i}=k T / 6 \pi r_{i} \eta$

yields effective radical radii of $r_{\mathrm{Fc}}=2.9 \AA$ and $r_{\mathrm{MB}}=5.8 \AA$ in fair agreement with the molecular dimensions of these compounds. Equating the reaction distance in the $\left(\mathrm{Fc}^{+} . . \mathrm{MB}\right)$ pair with the sum of these radii, i.e. with $r=8.7 \AA$, we obtain from Eq. (22)

$k_{\mathrm{ce}}=1.5 \times 10^{10} \mathrm{~s}^{-1}=15 \mathrm{~ns}^{-1}$

in excellent agreement with the value from the spin chemical simulation. In view of the model character of the calculation, the exact agreement with the spin chemical value must be considered as rather fortuitious and should not be overestimated.

A convenient possibility of determining the spin relaxation time $\tau_{\mathrm{s}}$ of $\mathrm{Fc}^{+}$by an independent method is through the $T_{1}$ time of the ${ }^{1} \mathrm{H}$ nuclei in the $\mathrm{Cp}$ ligands (cf. ref [23] for details of interrelation between nuclear and electronic spin relaxation in paramagnetic compounds). From inversion recovery experiments the $T_{1}$ time of $\mathrm{Fc}^{+}$protons in deuterated acetonitrile was found to be $0.657 \mathrm{~ms}$ [24]. Inserting this value together with the geometric data for the H-positions ( $2.86 \AA$ separation from the center at 54.0 degrees from the $g_{\|}$-axis [25]) into the generalized Solomon-Bloembergen equation given by Bertini et al. (cf. Eq. (11) in ref. [26]) yields a value of $5.7 \mathrm{ps}$ for the electron spin relaxation time, in fair agreement with the spin chemical result.

Altogether, the accuracy of the fit achieved in the spin chemical simulation and the independent confirmation of two of the parameter values determined through this fit, provides convincing evidence that the conclusions drawn from the present analysis are valid. Thus the high value found for $k_{\mathrm{bct}}$ the rate constant of (spin-allowed) backward electron transfer, corresponding to a time constant of about $1.5 \mathrm{ps,}$ must be considered as reliable. Certainly, such fast electron transfer processes are possible if the free energy of the reaction places it close to the activationless Marcus region and electron transfer rate constants of such order of magnitude have in fact been measured in linked donor-acceptor systems. However, they cannot be directly observed in systems where donor and acceptor have to find each other in free diffusion because in this case the formation of the radical pair is slower than its decay. Under such conditions of freely diffusing systems, the spin chemical method seems to be unique in providing absolute rate data for geminate processes.

\section{Conclusions}

The magnetic field effect on ferrocene photooxidation kinetics described in this letter represents a significant step in extending the applications of delectron spin chemistry as initiated with our studies on $\mathrm{Ru}$-trisbipyridyl type complexes to a larger group 
of transition metal compounds. Whereas in experiments with $\mathrm{Ru}$-trisbipyridyl complexes photoexcitation was effected through the metal complex, a triplet excited dye is used to produce the spin chemically active radical pair with a d-electron radical constituent in the present experiments. Since longlived photoreactive states suitable for intermolecular electron transfer - among which Ru-complexes figure so prominently - are the exception rather than the rule among transition metal complexes, the present method has a much wider potential for creating spin-chemically interesting radical pairs with paramagnetic components from the d-block elements.

The analysis of the magnetic field effect carried out in this work has, once more, demonstrated the validity of our spin chemical model wherein the electronic structure, the static and the dynamic magnetic properties of the d-electron system are quantitatively taken into account, in the reaction operator, the spin Hamiltonian and the relaxation operator, respectively. The determination of absolute values of rate constants of cage escape, backward electron transfer and fast paramagnetic relaxation attests to the practical value of this model for detailed kinetic studies.

\section{Acknowledgements}

We thank Ms. Monika Fahnenschmidt for measuring the ${ }^{1} \mathrm{H}-\mathrm{T}_{1}$-time of ferricenium. Financial support from the Volkswagenstiftung and the Fonds der Chemischen Industrie is gratefully acknowledged.

\section{References}

[1] U.E. Steiner and T. Ulrich, Chem. Rev. 89 (1989) 51; K.M. Salikhov, Yu.N. Molin, R.Z. Sagdeev, A.L. Buchachenko, Spin Polarization and Magnetic Field Effects in Radical Reactions, Elsevier: Amsterdam, The Netherlands, 1984.
[2] N.J. Turro, Pure\&Appl.Chem. 67 (1995) 199.

[3] K.A. McLauchlan and U.E. Steiner, Mol. Phys. 73 (1991) 241.

[4] H.-J. Wolff and U.E. Steiner, Z.Physik.Chem.N.F. 169 (1990) 147.

[5] U.E. Steiner and D. Bürßner, Z.Physik.Chem.N.F. 169 (1990) 159.

[6] D. Bürßner, H.-J. Wolff and U.E. Steiner, Z.Physik.Chem.N.F. 182 (1993) 297.

[7] D. Bürßner, H.-J. Wolff and U.E. Steiner, Angew. Chem. Int. Ed. 33 (1994) 1772.

[8] H.-J. Wolff, D. Bürßner and U.E. Steiner, Pure \& Appl Chem. 67 (1995) 167.

[9] A. Farmilo and F. Wilkinson, Chem.Phys.Letters 34 (1975) 575.

[10] S. Tamura, K. Kikuchi, H. Kokubun and A. Weller, Z.Physik.Chem.N.F. 121 (1980) 165.

[11] K. Bergmann and C.T. O'Konski, J. Phys.Chem. 67 (1963) 2169.

[12] H.-J. Wolff and U.E. Steiner, Z.Physik.Chem. 169 (1990) 147; T. Ulrich, U.E. Steiner and W. Schlenker, Tetrahedron 42 (1986) 6131.

[13] K. Kikuchi, S. Tamura, C. Iwanaga and H. Kokubun, Z.Phys.Chem.N.F. 100 (1977) 17.

[14] P. Kamat and N.N. Lichtin, J.Phys.Chem. 85 (1981) 814.

[15] U.E. Steiner and Yu.A. Serebrennikov, J.Chem.Phys. 100 (1994) 7503; Yu. A. Serebrennikov and U.E.Steiner, ibid. 7508.

[16] K.D. Warren, Structure and Bonding 27 (1976) 45.

[17] J.H. Ammeter, J.Magn.Res. 30 (1978) 299.

[18] J.H. Ammeter, N. Oswald and R. Bucher, Helv.Chim.Acta 58 (1975) 671 .

[19] P. Gilch, Diploma Thesis, University of Konstanz, 1995.

[20] M. Eigen, Z.Physik.Chem.N.F. 1 (1954) 176; M.Z.Hoffman, J.Phys.Chem. 92 (1988) 3458.

[21] T. Kuwana, and N. Winograd, J.Am.Chem.Soc. 93 (1971) 4343.

[22] K.L. Kostka and M.D. Radcliffe, J.Phys.Chem. 96 (1992) 2289.

[23] L. Banci, I. Bertini and C. Luchinat, Nuclear and Electron Relaxation, Verlag Chemie: Weinheim, 1991.

[24] P. Gilch, N. Lukzen, M. Fahnenschmidt, U.E. Steiner, to be published.

[25] E.F. Paulus, L. Schäfer, J. Organomet. Chem. 144 (1978) 205.

[26] I. Bertini, C. Luchinat and K.V. Vasavada, J.Magn.Res. 89 (1990) 243. 